\title{
The Expanded School Centre for Special Education. Madrid, Spain Invited Competition. First Prize. 2016
}

MARCOS PARGA

Syracuse University
A mass media channel is a transmission medium, a system in which information travels between an emitter and a receiver. The main broadcast media are writing, radio, television and internet, and are the "places" where opinions are exchanged, critical thinking is built, previous knowledge is transmitted and visual and textual contents are distributed. Therefore, we can assert that, for better or worse, mass media are able to represent and reflect on the world in which we live and the people who inhabit it.

When designing our proposal for the new Centre for Special Education in Madrid, our intention, beyond creating a building, has been to build a new "channel;." A space for exchange designed to explore and promote new educational trends, to enable experimental pedagogical strategies development and generate a suitable and unique environment that reinforces intellectual and physical growth of disabled children and children with special educational needs.

As a result, our building will perform as a kind of test pattern, a fixed sign that was displayed when the transmitter was active but waiting to broadcast a new program. The new School will operate as a base frame of open configuration pending of programming. Our School will be a new media channel waiting to be loaded with content and capable of supporting subsequent updates.

Throughout this process the main variable that finally drove our work arose when incorporating the reality of an uncertain future as a raw material. In order to deal with the unexpected, we focused on a sort of neutral architecture, repeatable and standardized (modular), released from the tyranny of the present and based on concepts such as unstable identity or programmed imperfection. The final goal is to design a building to-be-done, whose real construction will begin the opening day.

The New School therefore will be an urban scale building but with a certain rural component related to domesticated natures, an antidote to monotony, to immobility, to "comfort zones," becoming a catalyst for non-conformist and proactive attitudes. We seek for an environment where nothing is as it is expected to be (or maybe...), where thinking and doing differently, where being a student, teacher or neighbor involves participating, and where the prevailing heterogeneity will be able to accommodate any teaching strategy. The idea is to avoid predictability, designing an space to project ourselves into the unexpected and the unfinished that the user must discover.
This strategy will allow us to deal with a large and complex program for 1700 all-age pupils including, among other functions, secure lobby, parents room,large hall, multipurpose space, dining room, kitchen and formative kitchen, classrooms, early years rooms, life skills room, hydrotherapy pool, gym, physio room, psychomotor education room, winter garden, sensory garden, cropping areas, outdoor playgrounds, and sports facilities, and parking areas.

The new building will be built combining concepts such as adaptability, creative participation, continuous updating, domestication of scale, typological decoding, constructive standardization, spatial and functional recycling, urban interaction and progressive specialization, to shape to a new typology of educational environment: the EXPANDED SCHOOL. 


\section{The Expanded School}

Center tor Special Education. Maaridid.Spain
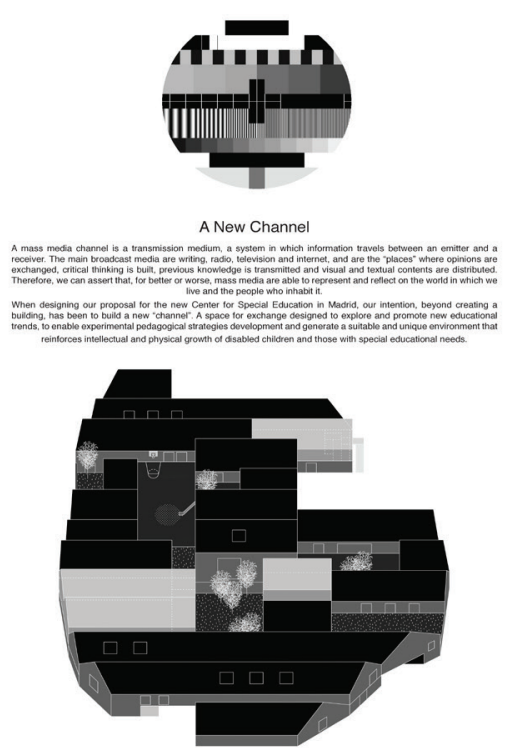

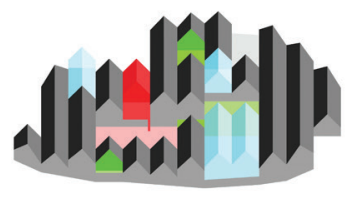

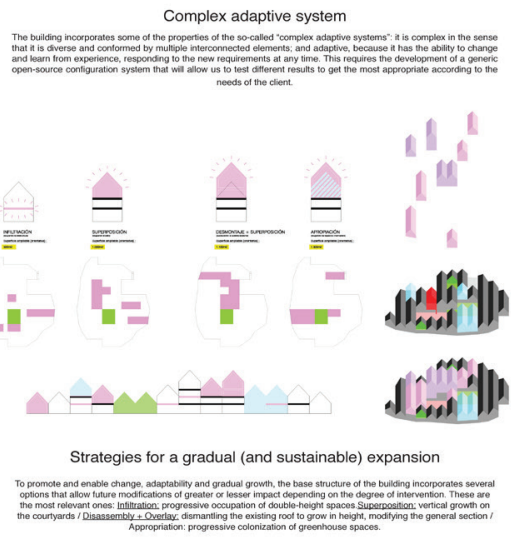$$
=\stackrel{=}{=}=-
$$
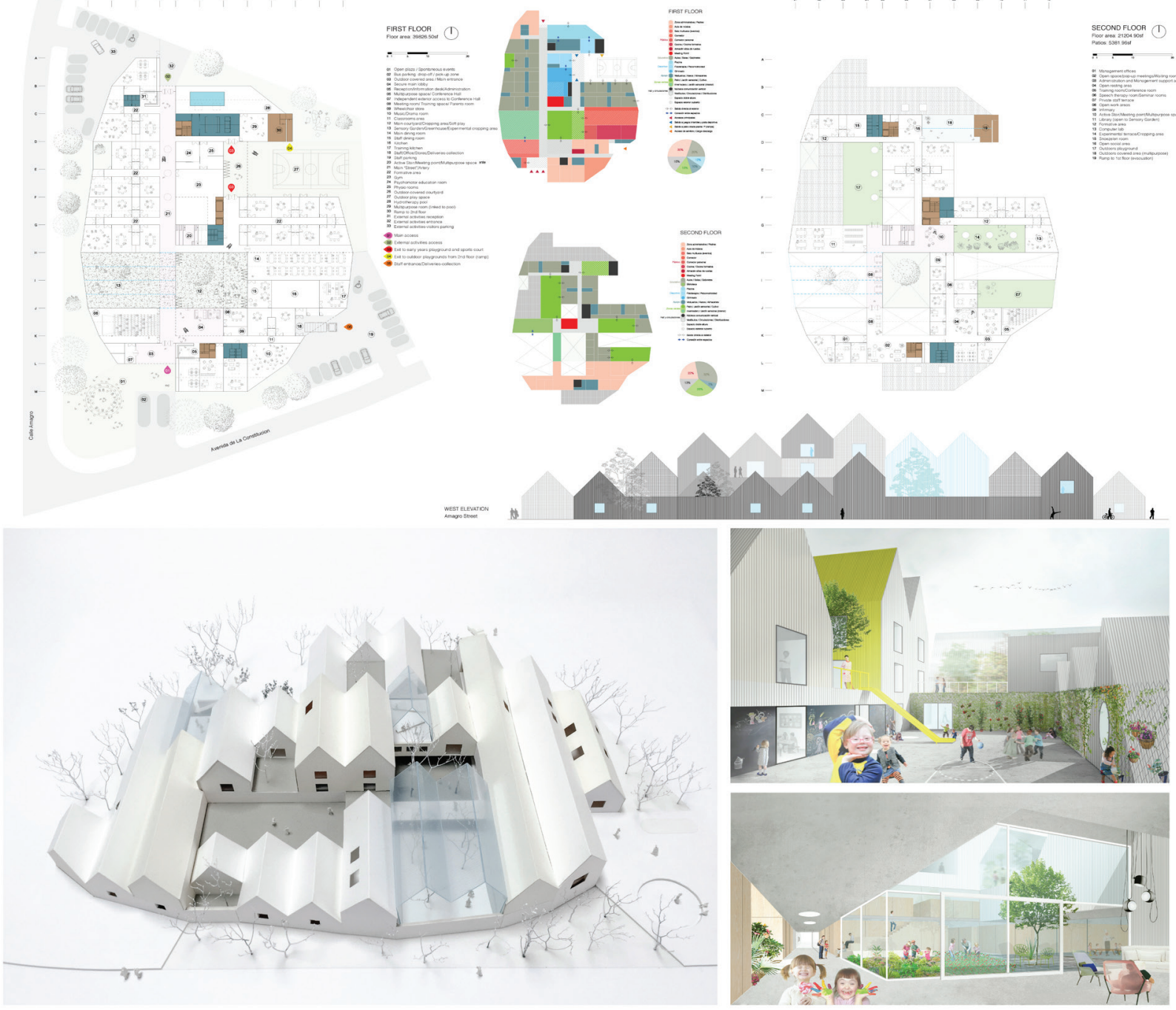